\title{
Commutator Anomalies and the Fock Bundle
}

Jouko Mickelsson

Department of Mathematics, University Jyväskylä, Seminaarinkatu 15, SF-40100, Jyväskylä, Finland

\begin{abstract}
We show that the anomalous finite gauge transformations can be realized as linear operators acting on sections of the bundle of fermionic Fock spaces parametrized by vector potentials, and more generally, by splittings of the fermionic one-particle space into a pair of complementary subspaces. On the Lie algebra level we show that the construction leads to the standard formula for the relevant commutator anomalies.
\end{abstract}

\section{Introduction}

We shall study the structure of the Fock bundle arising from a system of massless Weyl fermions coupled to an external non-Abelian Yang-Mills field in Hamiltonian framework. Let $M$ be the physical space of odd dimension. We are mainly concerned with the case $\operatorname{dim} M=3$ but we shall make some remarks about the (easier) case $\operatorname{dim} M=1$; our discussion of the case $\operatorname{dim} M=3$ can, without essential complications, be generalized to higher dimensions. We shall assume that $M$ is an oriented compact spin manifold with a given spin structure. Let $\mathscr{A}$ be the space of smooth $\mathbf{g}$ valued vector potentials on $M$, where $\mathbf{g}$ is the Lie algebra of a compact Lie group $G$.

Let $E$ be the tensor product (with a fixed inner product in the fibers) of the Dirac spin bundle and a trivial vector bundle over $M$, with a unitary representation $\rho$ of the gauge group $G$ in the fibers of the latter bundle. Let $H$ be the Hilbert space obtained as a completion from the space of smooth sections of $E$ with respect to the inner product

$$
\left(\psi, \psi^{\prime}\right)=\int_{M}\left\langle\psi(x), \psi^{\prime}(x)\right\rangle d(\text { vol })
$$

defined by a given volume form on $M$.

For each $A \in \mathscr{A}$ denote by $W_{A, \lambda}$ the plane in $H$ spanned by eigenvectors of the chiral massless Dirac (Weyl) operator $D_{A}$ corresponding to eigenvalues greater or equal to $\lambda \in \mathbb{R}$. Each plane $W=W(A, \lambda)$ determines a fermionic Fock space $\mathscr{F}_{W}$. The Fock space is generated from the vacuum by creation operators $a_{i}^{*}$ 
corresponding to an orthonormal basis in $W$ labelled by integers $i>0$, and by annihilation operators $a_{i}$ corresponding to an orthonormal basis in the complement $W^{\perp}$ labelled by integers $i \leqq 0$.

Note that in this way we obtain a bundle of Fock spaces labelled by the pairs $(A, \lambda)$ and not by the vector potentials $A$. But, since $\mathscr{A}$ is a vector space, we can always choose a plane $W(A)$ as a smooth function of $A \in \mathscr{A}$ such that $W(A)$ does not differ too much from any of the planes $W(A, \lambda), \lambda \in \mathbb{R}$; we shal explain this more precisely in the next section, but essentially "not too much" means that the different Fock representations of the canonical anticommutation relations are equivalent. However, it should be noted that there is no natural way to choose the function $W(A)$. On the other hand, the Fock representations corresponding to given $A$ but different $\lambda$ 's are naturally isomorphic up to a phase, [Se].

An important property of the planes $W(A, \lambda)$ is that they belong to an infinite-dimensional Grassmannian manifold $\mathrm{Gr}_{p}$ modelled by Schatten ideals $L_{2 p}$, where $2 p=1+\operatorname{dim} M$. Thus we are lead to examine the structure of a Fock bundle over the manifold $\mathrm{Gr}_{p}$. The Fock bundle over $\mathscr{A}$ is then obtained as a pull-back with respect to a chosen map $A \mapsto W(A)$. However, we shall see that there is a better way to define the Fock bundle over $\mathscr{A}$ which makes the construction independent of the choice of $W(A)$.

The action of the group $\mathscr{G}$ of gauge transformations on Dirac spinor field defines an action of $\mathscr{G}$ on the Grassmannian $\mathrm{Gr}_{p}$. The question is this: Can the action be lifted to the Fock bundle $\mathscr{F}$ over $\mathrm{Gr}_{p}$ ? We shall see in fact that there are two different natural candidates for the Fock bundle; the difference is that one of the bundles contains an everywhere nonvanishing vacuum section whereas the vacuum sector of the second bundle is twisted and there is no everywhere nonvanishing vacuum section. The origin for "commutator anomalies", or Schwinger terms, is the twisting of the vacuum section, [NA], in the second bundle: The group $\mathscr{G}$ acts only through a nontrivial Abelian extension $\hat{\mathscr{G}}$ in the Fock bundle. The Lie algebra of the extension in the case $\operatorname{dim} M=1$ is essentially an affine Kac-Moody algebra (central extension of the loop algebra $L_{g}$ ) whereas in higher dimensions one has operator valued Schwinger terms, [JJ, M1, F, Si].

The structure and consequences of the Schwinger terms in non-Abelian gauge theory in $3+1$ dimensions has been discussed, after the revival of the subject in [M1, F], in several papers in both space-time and Hamilton formulation; see, e.g., [BG, CS, DT, FHK, HS, J, NS, R, RSF, Se, Y], and references in those papers. The existence of this type of Schwinger terms was indicated in perturbative calculations already in $[\mathrm{JJ}]$.

The novel aspects of the present paper as compared to other discussions in the literature are the following. As already mentioned, we show that the appearance of the Schwinger terms can be understood in a simple "universal way" using the theory of infinite-dimensional Grassmannian manifolds. One might as well-study Fock space parametrized by other fields (e.g., a Higgs field) as long as the one-particle spaces are "comparable" (they are elements of the same Grassmannian $\mathrm{Gr}_{p}$ ). The second important feature is that we are able to show that the gague transformations, including the Schwinger terms, really act as well-defined operators between different fibers of a Fock bundle. In the earlier approaches the 
(generators of the) gauge transformations are well-defined in some chosen regularization and the Schwinger terms remain finite when the regularization is removed; however, the gauge operators themselves have not been defined as true Hilbert space transformations. Finally, we stress that our method is completely unperturbative.

We shall use the machinery of determinant bundles over infinite-dimensional Grassmannians developed in [PS] and later generalized in [MR] for discussing the commutator anomalies in $3+1$ space-time dimensions. Generalized Fock bundles were introduced in [M2] using the determinant bundle formalism for discussing the gauge group action; however, the Fock spaces studied in [M2] were not the standard ones of the canonical formalism. In this paper we want to show that essentially the same results can be obtained using the canonical formalism.

\section{Fock Bundle from Determinant Bundles}

Let $H$ be a complex separable Hilbert space (the one-particle space) and $H=H_{+} \oplus H_{-}$a splitting into a pair of closed infinite-dimensional subspaces. Fix an orthonormal basis $\left\{e_{n}\right\}_{n \in \mathbb{Z}}$ of $H$ such that $e_{n} \in H_{+}$for $n>0$ and $e_{n} \in H_{-}$for $n \leqq 0$. Denote by $\mathrm{Gr}_{p}(p=1,2, \ldots)$ the Grassmannian consisting of closed infinitedimensional subspaces $W \subset H$ such that

1. the projection $\mathrm{pr}_{\mathrm{H}_{+}}: W \rightarrow H_{+}$is a Fredholm operator,

2. the projection $\operatorname{pr}_{H_{-}}: W \rightarrow H_{-}$belongs to the Schatten ideal $L_{2 p}$.

The Schatten ideal $L_{2 p}$ consists by definition of those bounded operators $A$ for which $\left(A^{*} A\right)^{p}$ has a converging trace.

The manifold $\mathrm{Gr}_{p}$ is an union of disjoint connected components $\mathrm{Gr}_{p}^{(k)}(k \in \mathbb{Z})$ consisting of planes $W$ such that the Fredholm index dim ker-dim coker of the projection $\mathrm{pr}_{H_{+}}$is equal to $k$.

Each linear operator $g$ in $H$ can be written in the block form

$$
g=\left(\begin{array}{ll}
a & b \\
c & d
\end{array}\right)
$$

with respect to the splitting $H=H_{+} \oplus H_{-}$. Let $G L_{p}$ be the group of continuous invertible operators $g$ such that $b, c$ are in $L_{2 p}$. The group $G L_{p}$ acts in a natural way in $\mathrm{Gr}_{p}$; we can write $\mathrm{Gr}_{p}=G L_{p} / B_{+}$, where $B_{+}$consists of the operators $g$ which $c=0$.

A basis $w=\left\{w_{n}\right\}_{n=1,2, \ldots}$ of $W \in \mathrm{Gr}_{p}^{(0)}$ is said to be $p$-admissible (with respect to the basis $\left\{e_{n}\right\}_{n>0}$ of $\left.H_{+}\right)$if $w_{+}-1 \in L_{p}$, where $w_{+}$is the infinite matrix defined by

$$
\operatorname{pr}_{H_{+}} w_{i}=\sum_{j>0}\left(w_{+}\right)_{j i} e_{j}
$$

The set of all 1-admissible basis of all the planes $W \in \mathrm{Gr}_{p}^{(0)}$ forms an infinite-dimensional manifold $\mathrm{St}_{p}^{(0)}$. Any $W \in \mathrm{Gr}_{p}^{(0)}$ has such a basis, since a Fredholm operator of index zero is of the form invertible + finite rank operator. For the other components $\mathrm{Gr}_{p}^{(k)}$ the set of admissible basis is defined similarly except that 
$H_{+}$is replaced by the plane spanned by the vectors $e_{n}, n>-k$. (The manifold $\mathrm{St}_{p}$ defined here is different from the $\mathrm{St}_{p}$ employed in $[\mathrm{MR}]$.)

We recall from [PS] the geometric construction of the fermionic Fock space as the space of holomorphic sections of a complex line bundle DET* over $\mathrm{Gr}_{1}$. Let $G L^{1}$ be the group of invertible $\mathbb{N} \times \mathbb{N}$ matrices of the type $1+L_{1}$. A section of DET ${ }_{1}^{*}$ is by definition of a map $\psi: \mathrm{St}^{1} \rightarrow \mathbb{C}$ such that $\psi(w t)=\psi(w) \cdot \operatorname{det} t$ for each $t \in G L^{1}$. The vacuum is represented by the holomorphic section $\psi_{0}$ which is nonzero only on $\mathrm{St}_{1}^{(0)}$ and is there given by $\psi_{0}(w)=\operatorname{det} w_{+}$. A Fock basis is obtained as follows. Let $S=\left\{i_{1}, i_{2}, \ldots\right\}$ be an increasing sequence of integers such that $i_{n}-n=-k$ for $n \gg 0$. Denote by $\psi_{s}$ the section which is zero on $\mathrm{St}_{1}^{(m)}$ for $m \neq k$ and on $\mathrm{St}_{1}^{(k)}$ it is given by $\psi_{S}(w)=\operatorname{det} w_{S}$, where $w_{S}$ is the matrix obtained from the matrix $w_{i j}=\left\langle w_{j}, e_{i}\right\rangle$ by selecting rows labelled by the integers $S$. The inner product is defined such that the $\psi_{S}$ 's form an orthonormal basis. In a more standard language

$$
\psi_{S}=a_{j_{1}}^{*} \cdots a_{j_{s}}^{*} a_{i_{1}} \cdots a_{i_{r}}|\mathrm{vac}\rangle,
$$

where $j_{1}, \ldots, j_{s}$ are missing positive integers and $i_{1}, \ldots, i_{r}$ are the nonpositive integers in $S$.

We generalize the discussion above. Each plane $W \in \mathrm{Gr}_{p}(p \geqq 1)$ determines a Grassmannian $\mathrm{Gr}_{1}(W)$ consisting of planes $W^{\prime}$ such that

1. the projection of $W^{\prime}$ to $W^{\perp}$ is a Hilbert-Schmidt operator,

2. the projection of $W^{\prime}$ to $W$ is a Fredholm operator.

We define the Stiefel manifold $\mathrm{St}_{1}(W)$ for $W \in \mathrm{Gr}_{p}$ as follows. Choose a basis $w$ of $W$ such that $w \in \mathrm{St}_{p}$. A basis $w^{\prime}$ of a plane $W^{\prime} \in \mathrm{Gr}_{1}(W)$ belongs to $\mathrm{St}_{1}(W)$ if it is 1 -admissible with respect to $w$; this property does not depend on the choice of $w$.

The Fock space $\hat{\mathscr{F}}_{W}$ consists of complex valued holomorphic functions $\psi$ on $\mathrm{St}_{1}(W)$ transforming $\psi(w t)=\psi(w)$. det $t$ under a change $t \in G L^{1}$ of basis. The different Fock spaces form a Fock bundle $\hat{\mathscr{F}}$ over the base $\mathrm{Gr}_{p}$. A section of this bundle is a smooth map $\operatorname{Gr}_{p} \ni W \mapsto \psi_{W} \in \hat{\mathscr{F}}_{W}$, that is, $\psi$ is a function of $W \in \mathrm{Gr}_{p}$ and $f \in \operatorname{St}_{1}(W)$ such that $\psi_{W}(f t)=\psi_{W}(f) \cdot \operatorname{det} t$ for $t \in G L^{1}$. However, as we shall see, this is not the correct "physical" Fock bundle. The physical Fock bundle $\mathscr{F}$ is a tensor product of $\hat{\mathscr{F}}$ with a line bundle $\mathrm{DET}_{p}$ over $\mathrm{Gr}_{p}$, to be defined below.

Given an admissible basis $w$ of $W \in \mathrm{Gr}_{p}$ the Fock vacuum in $\hat{\mathscr{F}}_{W}$ is the holomorphic section $\psi(f)=\operatorname{det} f^{(w)}, f \in \operatorname{St}_{1}^{(0)}(W)$, where $f^{(w)}$ is the matrix representing the projection of $f$ to the basis $w$,

$$
f_{i}=\sum f_{j i}^{(w)} w_{j} \bmod W^{\perp} .
$$

The construction of the vacuum depends on the choice of the basis $w$ by a multiplicative factor $=\operatorname{det} t^{-1}$, where $t$ is a transformation of basis. This means that, although we have a well-defined vacuum line bundle $\operatorname{Vac}$ over $\mathrm{Gr}_{p}$, there is no everywhere nonvanishing global section of Vac.

The total space of the determinant bundle $\mathrm{DET}_{p}$ consists of all pairs $(w, \lambda) \in \mathrm{St}_{p} \times \mathbb{C}$ modulo the right action of the group $G L^{1}$ defined by

$$
(w, \lambda) \cdot t=\left(w t, \lambda \operatorname{det} t^{-1}\right) .
$$


A section is a complex valued function on $\mathrm{St}_{p}$ satisfying $\psi(w t)=\psi(w) \cdot \operatorname{det} t^{-1}$. The projection to the base $\mathrm{Gr}_{p}$ is given by $(w, \lambda) \mapsto \pi(w)$, where $\pi: \mathrm{St}_{p} \rightarrow \mathrm{Gr}_{p}$ is the canonical projection.

Let $\mathscr{F}=\hat{\mathscr{F}} \otimes \mathrm{DET}_{p}$. A section of $\mathscr{F}$ is then a complex valued function $\psi(f, w)$ of $w \in \mathrm{St}_{p}$ and $f \in \mathrm{St}_{1}(W)$, where $W$ is the linear span of $w$, such that

$$
\psi\left(f t, w t^{\prime}\right)=\psi(f, w) \cdot \operatorname{det} t \cdot \operatorname{det} t^{\prime-1}
$$

for $t, t^{\prime} \in G L^{1}$, and which is holomorphic in the variable $f . \mathscr{F}$ is the "physical" Fock bundle over $\mathrm{Gr}_{p}$. It has an everywhere nonvanishing vacuum section given by $\psi(f, w)=\operatorname{det} f^{(w)}$.

Let us now return to the problem of defining the Fock bundle over the space $\mathscr{A}$ of vector potentials. We set $2 p=1+\operatorname{dim} M$. Let now $W=W(A, \lambda)$ and $W^{\prime}=W\left(A, \lambda^{\prime}\right)$ for a given $A \in \mathscr{A}$ and for some $\lambda, \lambda^{\prime} \in \mathbb{R}$. We claim that

$$
\mathrm{St}_{1}(W)=\mathrm{St}_{1}\left(W^{\prime}\right) \text {. }
$$

Let $w, w^{\prime}$ be 1 -admissible basis of $W, W^{\prime}$, respectively. Since a product of operators of type $1+L_{1}$ is again in $1+L_{1}$, it is sufficient to show that $w^{\prime}$ is 1 -admissible relative to $w$. Suppose for example that $\lambda>\lambda^{\prime}$. Then $W^{\prime}=W \oplus V$, where $V$ is finite-dimensional. It follows that

$$
w_{i}^{\prime}=\sum_{j} \alpha_{j i} w_{j}+\sum_{j} \beta_{j i} w_{j}^{\perp},
$$

where $w^{\perp}$ is a basis of $W^{\perp}$ and $\beta$ is a matrix of finite rank. Then

Let $-k,-k^{\prime}$ be the Fredholm indices of the planes $W, W^{\prime}$, respectively.

$$
w_{i}=\sum_{j>k} a_{j i} e_{j}+\sum_{j \leqq k} b_{j i} e_{j}, \quad w_{i}^{\prime}=\sum_{j>k^{\prime}} a_{j i}^{\prime} e_{j}+\sum_{j \leqq k^{\prime}} b_{j i}^{\prime} e_{j} .
$$

The matrices $a, a^{\prime}$ belong to $1+L_{1}$ and $b, b^{\prime} \in L_{2 p}$. On the other hand, from (2.7) it follows that

$$
a^{\prime}=\alpha a+\text { a finite rank matrix. }
$$

Since both $a^{\prime}$ and $a$ are in $1+L_{1}$ it follows that $\alpha \in 1+L_{1}$.

Thus we have a well-defined Stiefel manifold $\mathrm{St}_{1}(A)=\mathrm{St}_{1}(W(A, \lambda))$ for each $A \in \mathscr{A}$ which does not depend on the choice of $\lambda$. The Fock space $\widehat{\mathscr{F}}_{A}$ is now defined as the space of holomorphic functions $\psi: \operatorname{St}_{1}(A) \rightarrow \mathbb{C}$ with $\psi(w t)=\psi(w) \cdot \operatorname{det} t$, $t \in G L^{1}$, in the same way as the Fock spaces $\widehat{\mathscr{F}}_{W}$ previously.

Given a map $A \mapsto W(A) \in \mathrm{Gr}_{p}$ we can pull back the determinant bundle over $\mathrm{Gr}_{p}$ to a line bundle over $\mathscr{A}$; taking a tensor product of this line bundle with the bundle of Fock spaces $\mathscr{F}_{A}$ we obtain a bundle of "physical" Fock spaces $\hat{\mathscr{F}}_{A}$ over $\mathscr{A}$; however, as already mentioned in the introduction, there is no natural way to choose the function $W(A)$.

\section{Gauge Group Action in Fock Bundles}

The group $G L_{p}$ does not act in $\mathrm{DET}_{p}$ : If $w \in \mathrm{St}_{p}$ then in general $g \cdot w$ is not in $\mathrm{St}_{p}$. However, we can always find a transformation $q=q(W)(W=\pi(w))$ of the basis 
such that $g w q^{-1} \in \mathrm{St}_{p}$. If $p=1$ then $q(W)$ can be chosen such that it depends only on $g$ and not on $W$. When $p>1 q(W)$ depends on $W$ also. If $q^{\prime}$ is another matrix with the same property as $q$ then $q^{\prime}=q t$ for some $t \in G L^{1}$. Let $(g, q, \lambda)$ be a triple consisting of $g \in G L_{p}$, of a function $q=q(W)$ such that $g w q(W)^{-1} \in \mathrm{St}_{p}$ for each $w \in \operatorname{St}_{1}(W)$, and a function $\lambda: \mathrm{Gr}_{p} \rightarrow \mathbb{C}^{\times}$. We define an action of $(g, q, \lambda)$ on $\mathrm{DET}_{p}$ by

$$
(g, q, \lambda) \cdot(w, \mu)=\left(g w q(W)^{-1}, \mu \lambda(W)\right),
$$

where $W$ is the plane spanned by $w$. These transformations form a group with the multiplication rule

$$
(g, q, \lambda) \cdot\left(g^{\prime}, q^{\prime}, \lambda\right)=\left(g g^{\prime}, q^{\prime \prime}, \lambda^{\prime \prime}\right)
$$

where $q^{\prime \prime}(W)=q\left(g^{\prime} W\right) q^{\prime}(W)$ and $\lambda^{\prime \prime}(W)=\lambda\left(g^{\prime} W\right) \lambda^{\prime}(W)$. The normal subgroup $N$ consisting of triples $(1, q(W)$, det $q(W))$, with $q: \mathrm{Gr}_{p} \rightarrow G L^{1}$, acts trivially on $\mathrm{DET}_{p}$ and therefore the group $\widehat{G L}_{p}$ obtained by taking the quotient of the whole group by $N$ acts on $\mathrm{DET}_{p}$.

The group $\widehat{G L}_{p}$ is a principal bundle over $\widehat{G L}_{p}$ with fiber $\operatorname{Map}\left(\mathrm{Gr}_{p}, \mathbb{C}^{\times}\right)$. The projection into the base is the mapping $(g, q, \lambda) \mapsto g . G L_{p}$ acts in $\widehat{\mathscr{F}}$ as follows. On the base $\mathrm{Gr}_{p}$ we have the natural action of $G L_{p}$. Let $\psi \in \widehat{\mathscr{F}}_{W}$ for some $W \in \mathrm{Gr}_{p}$. If $w \in \mathrm{St}_{1}(W)$ then $g w q(W)^{-1} \in \mathrm{St}_{1}(g W)$ for any $(g, q, \lambda) \in \widehat{G L}_{p}$ and therefore we can define an element $\psi^{\prime} \in \widehat{\mathscr{F}}_{g W}$ by

$$
\psi^{\prime}\left(w^{\prime}\right)=\psi\left(g^{-1} w^{\prime} q(W)\right) \cdot \lambda\left(g^{-1} W\right)^{-1},
$$

where $w^{\prime} \in \operatorname{St}_{1}(g W)$. This formula gives a homomorphism of $\widehat{G L}_{p}$ into the group of invertible bundle maps in $\hat{\mathscr{F}}$.

Let us compute explicitly the commutation relations of the Lie algebra extension $\widehat{g l}_{p}$ corresponding to the group $\widehat{G L}_{p}$ in the case $p=2, \operatorname{dim} M=3$. For that we need a more explicit form of the group law near the identity element. If $g \in G L_{2}$ is near 1 then the block $a$ has an inverse $a^{-1}$. For $W \in \mathrm{Gr}_{2}$ let $F: H \rightarrow H$ be the linear operator such that

$$
\left.F\right|_{W}=+1,\left.\quad F\right|_{W^{\perp}}=-1
$$

Writing

$$
F=\left(\begin{array}{ll}
F_{11} & F_{12} \\
F_{21} & F_{22}
\end{array}\right)
$$

with respect to the decomposition $H=H_{+} \oplus H_{-}$, the off-diagonal blocks belong to $L_{4}$ whereas $F_{11}-1 \in L_{2}, F_{22}+1 \in L_{2}$, [MR].

Lemma 3.6. Let $w \in \mathrm{St}_{1}(W), g=\left(\begin{array}{ll}a & b \\ c & d\end{array}\right), F$ the operator describing the plane $W \in \mathrm{Gr}_{2}$, and $q=a+\frac{1}{2} b F_{21}$. Then for $g$ in a small neighborhood of 1 (which does not depend on $W, w)$ the operator $q$ is invertible and $g w q^{-1}$ belongs to $\operatorname{St}_{1}(g W)$.

Proof. Since $F$ is unitary, the operator norm of $F_{21}$ is smaller or equal to one. Since $\|A\| \leqq\|A\|_{p}$ for $p \geqq 1$, the operator norm of $b F_{21}$ is small when $b$ is near zero in $L_{4}$ norm; on the other hand, $a$ is near 1 when $g$ is in a small neighborhood of the unity, and therefore $a+\frac{1}{2} b F_{21}$ is invertible when $g$ is near unity. 
For proving the second statement let us assume first that $w$ is an admissible basis of $W$. Now we have

$$
\left(g w q^{-1}\right)_{+}=\left(a w_{+}+b w_{-}\right) q^{-1}=a\left(w_{+}+a^{-1} b w_{-}\right)\left(1+\frac{1}{2} a^{-1} b F_{21}\right)^{-1} a^{-1} .
$$

This operator is in $1+L_{1}$ iff the operator $\left(1+a^{-1} b w_{-}\right)\left(1+\frac{1}{2} a^{-1} b F_{21}\right)^{-1}$ is $1+$ a trace class operator. When $b$ is small we can expand the second factor as a power series in $b$,

$$
\left(1+\frac{1}{2} a^{-1} b F_{21}\right)^{-1}=1-\frac{1}{2} a^{-1} b F_{21}+\cdots,
$$

where the rest is a trace class operator, since $b F_{21}$ is a Hilbert-Schmidt operator. Thus, modulo a trace class operator,

$$
\begin{aligned}
\left(1+a^{-1} b w_{-}\right)\left(1+\frac{1}{2} a^{-1} b F_{21}\right)^{-1} \\
\quad=1+a^{-1} b\left(w_{-}-\frac{1}{2} F_{21}\right)-a^{-1} b w_{-} \cdot \frac{1}{2} a^{-1} b F_{21}+\cdots .
\end{aligned}
$$

The last term is in $L_{1}$ since $w_{-}, b, F_{21} \in L_{4}$. In the second term $b\left(w_{-}-\frac{1}{2} F_{21}\right) \in L_{1}$ by a result in [MR].

Let then $w^{\prime} \in \mathrm{St}_{1}(W)$ be arbitrary. Let $\alpha$ be the matrix representing the projection of $w^{\prime}$ to the admissible basis $w$ of $W$. We have to show that $g w^{\prime} q^{-1}$ is admissible relative to $g w q^{-1}$. But the matrix representing the projection of the former to the latter is equal to $q \alpha q^{-1}$. Since $\alpha-1 \in L_{1}$, we have $q \alpha q^{-1} \in 1+L_{1}$, and so $g w^{\prime} q^{-1} \in \mathrm{St}_{1}(W)$.

From the lemma follows especially that $(g, q, 1)$ belongs to $\widehat{G L}_{p}$ when $g$ is near 1 and $q=q(W)$ is given by the lemma. Let $g_{1}, g_{2} \in G L_{2}$ and denote by $q_{12}$ the operator valued function corresponding to the product $g_{1} g_{2}$. We can write

$$
\left(g_{1}, q_{1}, 1\right)\left(g_{2}, q_{2}, 1\right)=\left(g_{1} g_{2}, q, 1\right) \equiv\left(g_{1} g_{2}, q_{12}, \omega\left(g_{1}, g_{2}\right)\right) \bmod N
$$

where $q(W)=q_{1}\left(g_{2}^{-1} W\right) q_{2}(W)$ and $\omega\left(g_{1}, g_{2}\right)$, a local 2-cocycle on $G L_{2}$, is a $\mathbb{C}^{\times}$ valued function on $\mathrm{Gr}_{2}$, given by

$$
\begin{aligned}
\omega\left(g_{1}, g_{2}\right)(W)= & \operatorname{det}\left\{\left[a_{1} a_{2}+b_{1} c_{2}+\frac{1}{2}\left(a_{1} b_{2}+b_{1} d_{2}\right) F_{21}\right]\right. \\
& \cdot\left(a_{2}+\frac{1}{2} b_{2} F_{21}\right)^{-1} 2\left(2 a_{1}+b_{1} c_{2} F_{11} \alpha_{2}+b_{1} c_{2} F_{12} \gamma_{2}\right. \\
& \left.\left.+b_{1} d_{2} F_{21} \alpha_{2}+b_{1} d_{2} F_{22} \gamma_{2}\right)^{-1}\right\},
\end{aligned}
$$

where

$$
\left(\begin{array}{ll}
\alpha_{2} & \beta_{2} \\
\gamma_{2} & \delta_{2}
\end{array}\right)=g_{2}^{-1} .
$$

For any Lie group the commutator of a pair $X, Y$ of Lie algebra elements is obtained from

$$
\left[X_{1}, X_{2}\right]=\left.\frac{1}{2} \frac{d^{2}}{d t d s} e^{t X_{1}} e^{s X_{2}} e^{-t X_{1}} e^{-s X_{2}}\right|_{s=t=0},
$$

and therefore the Lie algebra cocycle is

$$
c\left(X_{1}, X_{2}\right)=\left.\frac{d^{2}}{d t d s} \omega\left(e^{t X_{1}}, e^{s X_{2}}\right)\right|_{s=t=0} .
$$


When $X_{i}=\left(\begin{array}{ll}a_{i} & b_{i} \\ c_{i} & d_{i}\end{array}\right)$, we obtain

$$
\begin{aligned}
c\left(X_{1}, X_{2}\right) & =\operatorname{tr}\left(b_{1} c_{2}-b_{2} c_{1}+\frac{1}{2} b_{2} c_{1} F_{11}-\frac{1}{2} b_{1} c_{2} F_{11}+\frac{1}{2} c_{2} b_{1} F_{22}-\frac{1}{2} c_{1} b_{2} F_{22}\right) \\
& =\frac{1}{8} \operatorname{tr}(F-\varepsilon)\left[\left[\varepsilon, X_{1}\right],\left[\varepsilon, X_{2}\right]\right]
\end{aligned}
$$

where $\varepsilon=\left(\begin{array}{rr}1 & 0 \\ 0 & -1\end{array}\right)$. This cocycle was derived earlier in [MR] using a different approach. We have now proven the following theorem:

Theorem 3.13. The group $\mathrm{GL}_{2}$ acts in the bundle $\mathrm{DET}_{2}$ over $\mathrm{Gr}_{2}$ such that the action on the base $\mathrm{Gr}_{2}$ is the natural action of $G L_{2}$. The Lie algebra $\widehat{\mathbf{g}}_{2}$ of $\widehat{G L}_{2}$, which as a vector space is the direct sum of $\mathbf{g l}_{2}$ with the Abelian Lie algebra $\mathrm{Map}\left(\mathrm{Gr}_{2}, \mathbb{C}\right)$, is defined by the commutator

$$
\left[\left(X_{1}, \lambda_{1}\right),\left(X_{2}, \lambda_{2}\right)\right]=\left(\left[X_{1}, X_{2}\right], X_{1} \cdot \lambda_{2}-X_{2} \cdot \lambda_{1}+c\left(X_{1}, X_{2}\right)\right),
$$

where $X \cdot \lambda$ is the Lie derivative of the function $\lambda$ in the direction of the vector field $X$ on the manifold $\mathrm{Gr}_{2}$ (with respect to the canonical action of $G L_{2}$ on $\mathrm{Gr}_{2}$.)

The cocycle (3.12) is a "universal cocycle" corresponding to the 2-cocycle

$$
c_{0}(X, Y, A)=\text { const. } \int_{M} \operatorname{tr}(X d Y-Y d X) \wedge d A
$$

in three space dimensions, where $X, Y: M \rightarrow \mathbf{g}$ are infinitesimal gauge transformations and $A \in \mathscr{A}$, see [M1] and [F].

In the case $p=1$, corresponding to $\operatorname{dim} M=1$, we can set $q(W)=a$ in the computations above and the cocycle is in that case simply

$$
-\frac{1}{8} \operatorname{tr} \varepsilon\left[\left[\varepsilon, X_{1}\right],\left[\varepsilon, X_{2}\right]\right]=\operatorname{tr}\left(b_{1} c_{2}-b_{2} c_{1}\right),
$$

which was derived in [L], [PS].

We are now able to discuss the action of $G L_{2}$ in the Fock bundles $\mathscr{F}$ and $\hat{\mathscr{F}}$. Let $\psi=\psi(f, w)$ be a section of $\mathscr{F}$. For $g \in G L_{2}$ define $\psi^{\prime}=T(g) \psi$ by

$$
\psi^{\prime}(f, \omega)=\psi\left(g^{-1} f q(W), g^{-1} w q(W)\right),
$$

where $W=\pi(w)$ and $q$ is chosen such that $g^{-1} w q$ is admissible. The section $\psi^{\prime}$ does not depend on the choice of $q$ by the formula (2.5). Thus $G L_{2}$ acts properly, without any projective factors, in $\mathscr{F}$. However, this is not so for the bundle $\hat{\mathscr{F}}$. By definition of $\mathscr{F}, \hat{\mathscr{F}}=\mathscr{F} \otimes \mathrm{DET}_{2}^{*}$, and therefore we have a natural action of $\widehat{G L}_{2}$, not of $G L_{2}$, in the bundle $\hat{\mathscr{F}}$.

Theorem 3.16. The group $\widehat{G L}_{2}$ acts in the Fock bundle $\widehat{\mathscr{F}}$ such that the action on the base is the canonical action of $\mathrm{GL}_{2}$ and the corresponding Lie algebra cocycle (Schwinger term) is $(-1) \times$ the cocycle (3.12).

Finally we shall discuss the action of the gauge group $\mathscr{G}$ in the Fock bundle $\hat{\mathscr{F}}$ over $\mathscr{A}$, as defined in the Sect. 2. The group $\mathscr{G}$ acts in $H$ through pointwise multiplication of spinor fields. It is known that $\mathscr{G} \subset G L_{2}$, when the space $M$ is three-dimensional, see, e.g., [MR]. The extension $\widehat{G L}{ }_{2}$ of $G L_{2}$ defines thus also an 
Abelian extension $\hat{\mathscr{G}}$ of $\mathscr{G}$. The action in the Fock bundle is defined in a similar way as was done in the case of Fock spaces parametrized by elements of $\mathrm{Gr}_{2}$.

Let $g \in \mathscr{G}$. For $A \in \mathscr{A}$ we can choose an infinite matrix $q(A)$ such that $g w q(A)^{-1} \in \mathrm{St}_{1}\left(A^{g^{-1}}\right)$ for $w \in \mathrm{St}_{1}(A)$, where $A^{g}=g^{-1} A g+g^{-1} d g$. The action of a triple $(g, q, \lambda)$, where $\lambda: \mathscr{A} \rightarrow \mathbb{C}^{\times}$, on sections $\psi(A, w)$ of the Fock bundle is then given by

$$
[T(g, q, \lambda) \psi](A, w)=\lambda\left(A^{g}\right)^{-1} \psi\left(A^{g}, g^{-1} w q(A)\right) .
$$

The normal subgroup consisting of triples $(1, q$, det $q)$, where $q: \mathscr{A} \rightarrow G L^{1}$, acts trivially in $\hat{\mathscr{F}}$ and therefore we have an action of the quotient group $\hat{\mathscr{G}}$ in $\widehat{\mathscr{F}}$. The group $\hat{\mathscr{G}}$ is a principal bundle with fiber $\operatorname{Map}\left(\mathscr{A}, \mathbb{C}^{\times}\right)$over $\mathscr{G}$. The group $\operatorname{Map}\left(\mathscr{A}, \mathbb{C}^{\times}\right)$is an Abelian normal subgroup of $\hat{\mathscr{G}}$.

Of course, the action of $\mathscr{G}$ in the bundle $\mathscr{F}$ over $\mathscr{A}$ does not involve any commutator anomally, but, moving from $\widehat{\mathscr{F}}$ to $\mathscr{F}$ requires a choice of the function $W(A)$.

\section{References}

[BG] Banerjee, R., Ghosh, S.: The Gauss operator in anomalous gauge theories: A Hamiltonian formulation. Modern Phys. Lett. A4, 855 (1989)

[CS] Cabra, D., Schaposnik, F. A.: On regularization ambiguities in fermionic models. J. Math. Phys. 30, 816 (1989)

[DT] Dunne, G. V., Trugenberg, C. A.: Kinetic normal ordering and the Hamiltonian structure of $U(1)$ Chiral anomalies in $3+1$ dimensions. Preprint CTP-1708, M.I.T., Mass., 1989

[F] Faddeev, L.: Operator anomaly for the Gauss law. Phys. Lett. 145B, 81 (1984); Faddeev, L., Shatasvili, S. L.: Theor. Math. Phys. 60, 770 (1984)

[FHK] Fujiwara, T., Hosono, S., Kitakado, S.: Chirally Gauged Wess-Zumino-Witten models as constraint system. Modern Phys. Lett. A3, 1585 (1988)

[HS] Hosono, S., Seo, K.: Derivation of chiral anomalies and commutator anomalies in a fixed time regularization method. Phys. Rev. D38, 1296 (1988)

[HT] Harada, K., Tsutsui, I.: A consistent Gauss law in anomalous gauge theory. Progr. Theor. Phys. 78, 675 (1987)

[J] Jo, S.-C.: Commutator of gauge generators in non-Abelian chiral theory. Nucl. Phys. B256, $616(1985)$

[JJ] Jackiw, R., Johnson, K.: Anomalies of the axial vector current. Phys. Rev. 182, 1459 (1969)

[KY] Kolokolov, I. V., Yelkhovsky, A. S.: Schwinger terms as a source of gauge anomaly in Hamiltonian approach. Preprint, Inst. of Nuclear Physics, Novosibirsk 1987

[L] Lundberg, Lars-Erik: Quasi-Free Second Quantization. Commun. Math. Phys. 50, 103 (1976)

[M1] Mickelsson, J.: Chiral anomalies in even and odd dimensions. Commun. Math. Phys. 97, 361 (185); On a relation between massive Yang-Mills theories and dual string models. Lett. Math. Phys. 7, 45 (1983); Kac-Moody groups, topology of the Dirac determinant bundle, and fermionization. Commun. Math. Phys. 110, 173 (1987)

[M2] Mickelsson, J.: Current Algebras and Groups. London, New York: Plenum Press (in press); current algebra representation for the $3+1$ dimensional Dirac-Yang-Mills theory. Commun. Math. Phys. 117, 261 (1988)

[MR] Mickelsson, J., Rajeev, S.: Current algebras in $d+1$ dimensions and determinant bundles over infinite-dimensional Grassmannians. Commun. Math. Phys. 116, 365 (1988)

[NA] Nelson, P., Alvarez-Gaumé, L.: Hamiltonian Interpretation of Anomalies. Commun. Math. Phys. 99, 103 (1985)

[NS] Niemi, A., Semenoff, G.: Quantum holonomy and the chiral gauge anomaly. Phys. Rev. Lett. 55, 927 (1985) 
[PS] Pressley, A., Segal, G.: Loop Groups. Oxford: Clarendon Press 1986

[R] Rajeev, S.: Fermions from bosons in $3+1$ dimensions through anomalous commutators. Phys. Rev. D29, 2944 (1984)

[RSF] Reiman, A. C., Semenov-Tyan-Shanskii, M. A., Faddeev, L. D.: Quantum anomalies and cocycles on gauge groups. J. Funct. Anal. Appl. 18, 319 (1985)

[Se] Segal, G.: Faddeev's anomaly in Gauss' law. Preprint (unpublished). Department of Math., Oxford University 1985

[Si] Singer, I.: Families of Dirac operators with applications to physics. Asterisque 323, 1985

[Y] Yamagishi, H.: A space-time approach to chiral anomalies. Preprint, Department of Physics, State University of New York at Stony Brook 1987

Communicated by L. Alvarez-Gaumé

Received May 17, 1989 